\title{
Acupuncture for migraine prophylaxis: a randomized controlled trial
}

\author{
Ying Li MD PhD, Hui Zheng MD PhD, Claudia M. Witt MD MBA, Stephanie Roll PhD, Shu-guang Yu MD, Jie \\ Yan MD, Guo-jie Sun MD, Ling Zhao MD, Wen-jing Huang MD, Xiao-rong Chang MD, Hong-xing Zhang MD \\ PhD, De-jun Wang MD, Lei Lan MD, Ran Zou MD, Fan-rong Liang MD
}

See related commentary by Molsberger and colleagues on page 391 and at www.cmaj.ca/lookup/doi/10.1503/cmaj.112032 and research article by Smelt and colleagues on page E224 and at www.cmaj.ca/lookup/doi/10.1503/cmaj.110908

\section{- Abstract}

Background: Acupuncture is commonly used to treat migraine. We assessed the efficacy of acupuncture at migraine-specific acupuncture points compared with other acupuncture points and sham acupuncture.

Methods: We performed a multicentre, singleblind randomized controlled trial. In total, 480 patients with migraine were randomly assigned to one of four groups (Shaoyangspecific acupuncture, Shaoyang-nonspecific acupuncture, Yangming-specific acupuncture or sham acupuncture [control]). All groups received 20 treatments, which included electrical stimulation, over a period of four weeks. The primary outcome was the number of days with a migraine experienced during weeks 58 after randomization. Our secondary outcomes included the frequency of migraine attack, migraine intensity and migrainespecific quality of life.

Results: Compared with patients in the control group, patients in the acupuncture groups reported fewer days with a migraine during weeks $5-8$, however the differences between treatments were not significant $(p>0.05)$. There was a significant reduction in the number of days with a migraine during weeks $13-16$ in all acupuncture groups compared with control (Shaoyang-specific acupuncture v. control: difference -1.06 [95\% confidence interval $(\mathrm{Cl})$ -1.77 to -0.5 ], $p=0.003$; Shaoyang-nonspecific acupuncture v. control: difference $-1.22[95 \% \mathrm{Cl}$ -1.92 to -0.52 ], $p<0.001$; Yangming-specific acupuncture v. control: difference $-0.91[95 \% \mathrm{Cl}$ -1.61 to -0.21 ], $p=0.011$ ). We found that there was a significant, but not clinically relevant, benefit for almost all secondary outcomes in the three acupuncture groups compared with the control group. We found no relevant differences between the three acupuncture groups.

Interpretation: Acupuncture tested appeared to have a clinically minor effect on migraine prophylaxis compared with sham acupuncture.

Trial Registration: Clinicaltrials.gov NCT00599586
Competing interests: None declared.

This article has been peer reviewed.

Ying Li and Hui Zheng contributed equally to this article.

Correspondence to: Dr. Fan-rong Liang, acuresearch@126.com

CMAJ 2012. DOI:10.1503 /cmaj.110551
A bout $6 \%-8 \%$ of men and $16 \%-18 \%$ of women in the United States and England experience migraines, with or without an aura. ${ }^{1,2} \mathrm{~A}$ prevalence of $1 \%$ has been reported in mainland China, ${ }^{3}$ compared with $4.7 \%$ in Hong Kong and $9.1 \%$ in Taiwan. ${ }^{4.5} \mathrm{~A}$ recent Cochrane meta-analysis suggests that acupuncture as migraine prophylaxis is safe and effective and should be considered as a treatment option for willing patients. ${ }^{6}$

Although the specific effects acupuncture are controversial, acupuncture, as it is currently practised, clearly differentiates between real acupuncture points and nonacupuncture points. The Chinese Government launched the National Basic Research Program to obtain more data about the specificity of acupuncture points. ${ }^{?}$
Trials from Italy and Brazil ${ }^{8,9}$ showed that acupuncture was more effective than sham acupuncture in preventing migraines, but other trials have reported no differences..$^{10-13}$ There is no evidence that one acupuncture strategy is more effective than another for treating migraines. According to acupuncture theory, a headache on the lateral side is usually defined as a Shaoyang headache. In Jinkuiyi,${ }^{14}$ migraines are said to affect the yang meridians (including the Taiyang, Yangming and Shaoyang meridians). In Lingshu, ${ }^{15}$ the Shaoyang meridians are said to go through the lateral side of the body, therefore the Shaoyang meridians are thought to be superior for treating migraines. Some points on the Shaoyang meridians are regarded as being more specific for migraines than other points. ${ }^{16}$ 
Our aim was to investigate whether acupuncture at specific acupuncture points was more efficacious in preventing migraine than sham acupuncture at nonacupuncture points. We also investigated whether the efficacy varied when acupuncture points along different meridians or points along the same meridian were used.

\section{Methods}

\section{Study design}

We performed a multicentre, single-blind, randomized controlled trial with four arms: three acupuncture groups (Shaoyang-specific, Shaoyang-nonspecific and Yangming-specific acupuncture) and one sham acupuncture (control) group. We followed the guidelines of the International Headache Society for clinical trials involving patients with migraines. ${ }^{17}$ Our trial was carried out in nine hospitals in China from April 2008 to December 2009, with a four-week baseline period followed by randomization.

\section{Ethics approval}

The trial protocol was approved by all local institutional ethics review boards and follows the principles of the Declaration of Helsinki and the Chinese version of the International Conference on Harmonisation - Good Clinical Practice, including onsite monitoring ${ }^{18}$ and training of investigators. ${ }^{19}$

All patients gave written informed consent. The trial was registered (clinicaltrials.gov: NCT00599586), and the design has been prepublished. $^{20}$

\section{Setting and participants}

Patients were recruited through hospital-based recruitment and television and newspaper advertisements. They had to meet the International Headache Classification's criteria for migraines with or without an aura. ${ }^{21}$ We included people who met the following criteria: experienced acute migraine attacks for more than one year with two or more attacks per month during the previous three months and during the baseline period; aged 18-65 years; onset of migraines before age 50; completed a baseline headache diary; did not take any prophylactic migraine medication during the previous month; willing to complete 20 acupuncture treatments during a four-week period (weeks $1-4)$; and able to provide written informed consent. We excluded patients who had headache due to organic disorders (e.g. subarachnoid hemorrhage, cerebral hemorrhage, cerebral embolism, cerebral thrombosis, vascular malformation, arthritis, hypertension, arteriosclerosis), psychosis, pregnancy or lactation, allergies, bleeding disorders or serious diseases of the heart, liver, kidney or other organs.

\section{Randomization and interventions}

Randomization was performed by the National Clinical Trial Center of Chinese Medicine, Chengdu Good Clinical Practice Center. Central randomization was performed by text messages sent by the investigator or by use of a website and email confirmation. The randomization sequence (blocked, stratified for centres) was generated by use of the randomization module of the synthesized management platform of the Chengdu Good Clinical Practice Centre (block length 12, unknown to centres). Patients, outcome assessors and statisticians were blinded as to randomization. Patients were informed that they would receive one of four types of acupuncture treatment, three of which used traditional Chinese acupuncture theories and one which was based on modern acupuncture theory.

The treatments, which included electrostimulation, were provided by specialized acupuncturists who had at least five years' training and five years' experience using a standardized protocol (Table 1). We selected the acupuncture points according to a systematic review of ancient and modern literature, ${ }^{16,22}$ consensus meetings with experts and experience from our previous study. ${ }^{23}$ The Shaoyang-specific and sham acupuncture points chosen were used in a previous study of acute migraine attacks. ${ }^{23}$

Acupuncture was applied unilaterally, alternating between the left and right sides. The goal was to elicit a de qi sensation (a range of sensations typically generated by the insertion of a needle into an acupuncture point and the manipulation of the needle) in the three acupuncture groups but not in the sham-acupuncture group. Two types of Hwato needles (Suzhou Hua Tuo Medical Instruments, Suzhuo, China) were used in all groups (length 25-40 mm, diameter $0.25 \mathrm{~mm}$; length 13 $\mathrm{mm}$, diameter $0.18 \mathrm{~mm}$ ). The patients received 20 treatments (30 min each) over a four-week period: once per day for five consecutive days followed by a two-day break. The details of this procedure have been published. ${ }^{19}$

The patients were instructed not to take any regular medications for the treatment of migraines. In cases of severe pain, ibuprofen (300 mg each capsule with sustained release) was allowed as rescue medication.

\section{Outcomes}

Our primary outcome was the number of days with a migraine, as recorded by participants in a diary, during a four-week period after acupuncture was given (weeks 5-8 after randomization). 
Patients were given an explanation of what to classify as a migraine-specific headache. Our secondary outcomes, also obtained from the patients' diaries, were frequency of migraines (defined as the number of migraines separated by pain free intervals of at least $48 \mathrm{~h}$ ), intensity of the migraine on a scale of $0-3$, and intensity of pain on a visual analogue scale from $0-10$.

Patients completed the diaries during four periods: baseline ( 4 wks before treatment), treatment (weeks 1-4) and follow-up (weeks 5-8 and 13-16). If a patient took medication for a migraine attack, the patient was asked to document the name, dose and time of intake; they were also asked to record when the pain subsided and any side effects experienced. We measured migraine-specific quality of life using the Migraine-Specific Quality-of-Life Questionnaire ${ }^{24,25}$ at baseline and at weeks 4,8 and 16 . We considered differences in quality of life to be clinically important if there were differences of at least 3.2, 4.6 and 7.5 points between groups for the subscales "role restrictive," "role preventive" and "emotional functional," respectively. ${ }^{26}$ We documented any adverse events, dropouts and reasons during the trial period.

\section{Statistical analysis}

To have $90 \%$ power (5\% significance level, twosided) to detect a difference of 1.6 migraine days between the Shaoyang-specific acupuncture and control groups, 105 patients per group were required. ${ }^{19}$ We assumed a standard deviation of 2.4 days for the sham acupuncture group and 4.0 days for the Shaoyang-specific group and an improvement of 2.4 and 4.0 days for these two groups. To account for dropouts, we recruited 120 patients per group (480 total).

Before the analyses were performed, a detailed statistical analysis plan was created and signed by the people responsible. The intentionto-treat population was defined as the number of patients assigned to treatment who received at least one treatment session. The per-protocol subgroup was the number of patients who completed the study without major protocol violation. All analyses were based on the intention-totreat population unless otherwise stated.

To account for multiplicity (comparison of three treatment groups with one control group), we used a three-step hierarchical testing procedure for confirmatory analysis of the primary outcome (analysis of covariance adjusted for centre

Table 1: Protocol for acupuncture for migraine prophylaxis

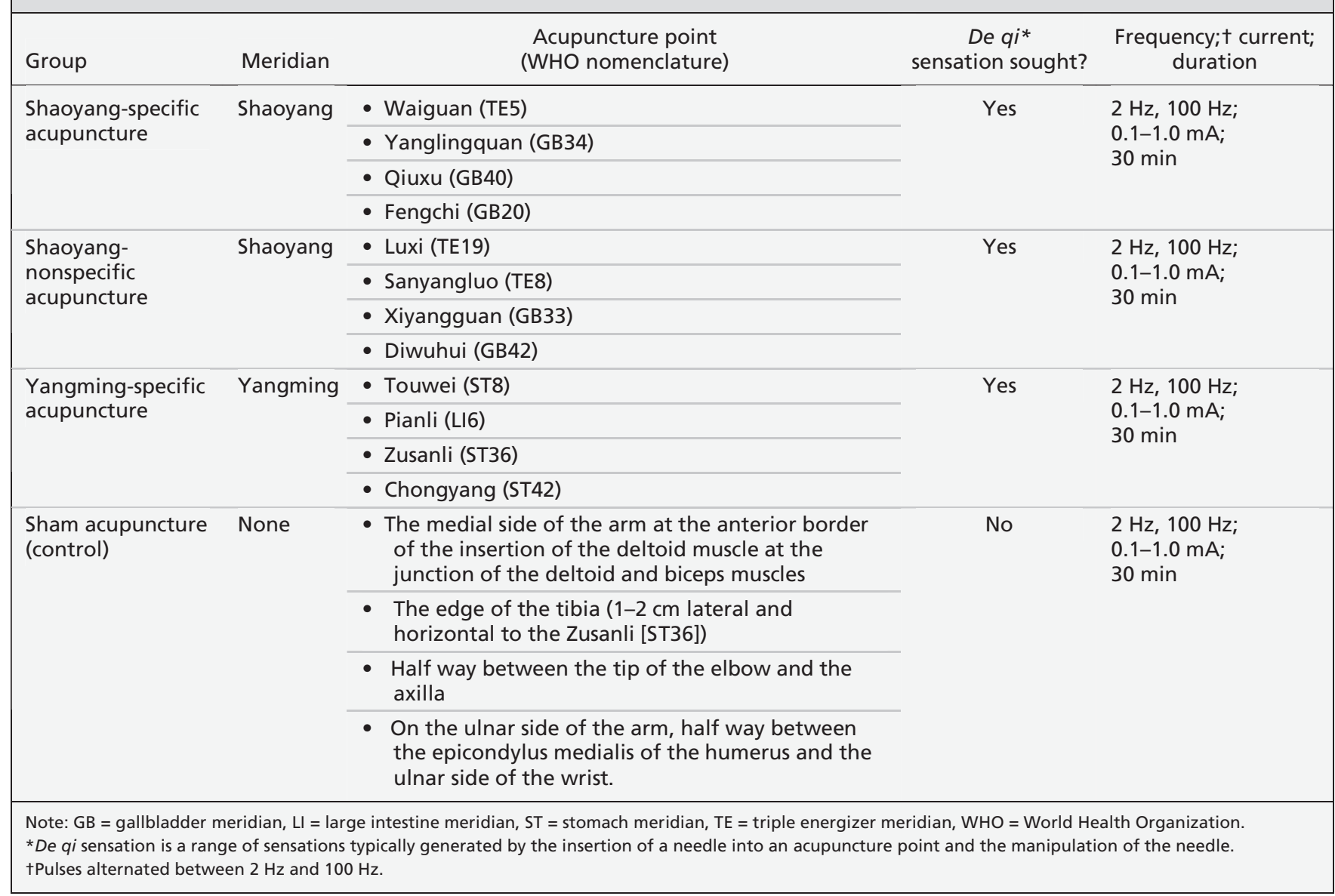


and baseline values) to compare the Shaoyangspecific (step one), Shaoyang-nonspecific (step two) and Yangming-specific (step three) acupuncture groups with the control group. If the difference was significant (5\% level, two-sided), the subsequent step was performed (otherwise, the subsequent $p$ values were considered explorative).

For the sensitivity analyses, we analyzed the primary outcome using two types of imputation for missing values for the intention-to-treat population: last observation carried forward and multiple imputation (maximum-likelihood-based regression method). We also analyzed the pri- mary outcome in the per-protocol population.

We analyzed the secondary outcomes by analysis of covariance or Poisson regression adjusted for the centre and baseline value. This analysis was based on the intention-to-treat population.

\section{Results}

\section{Participants}

After screening 1920 patients, 480 were randomly assigned to treatment between April 1, 2008, and August 12, 2009. In total, 476 patients received acupuncture treatment and were included in the

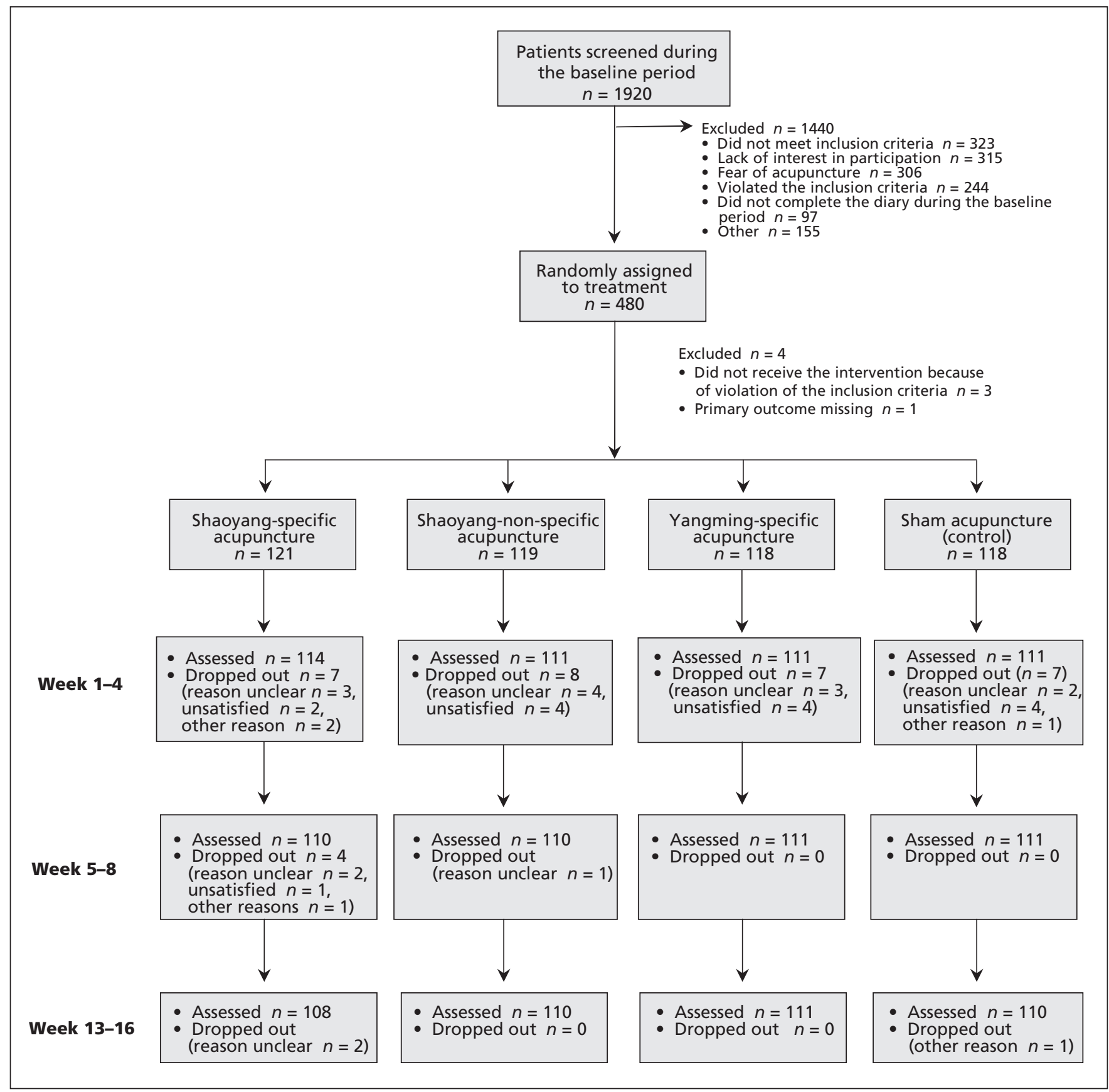

Figure 1: Flow chart of trial participants. Treatment was given during weeks 1-4; outcomes were assessed during week 4, 8 and 16. 
intention-to-treat analysis (Figure 1). The baseline parameters did not differ significantly between groups (Table 2). A total of 37 participants were lost at the end of the follow-up period (13 in the Shaoyang-specific acupuncture group, 9 in the Shaoyang-nonspecific acupuncture group, 7 in the Yangming-specific acupuncture group and 8 in the control group). In total, $423(88.9 \%)$ patients received at least 16 acupuncture treatments (108 in the Shaoyang-specific acupuncture group, 102 in the Shaoyang-nonspecific acupuncture group, 106 in the Yangming-specific acupuncture group and 107 in the control group).

\section{Efficacy}

We found no significant differences between any of the three acupuncture groups compared with sham acupuncture (control) for the number of days with a migraine during the four-week period after treatment (weeks 5-8; Table 3, Figure 2). We also found no differences between the three acupuncture groups (Shaoyang-specific acupuncture v. Shaoyang-nonspecific acupuncture, $p=0.96$; Shaoyang-specific acupuncture v. Yangming-specific acupuncture, $p=0.74$;
Shaoyang-nonspecific acupuncture v. Yangmingspecific acupuncture, $p=0.71$ ).

The frequency and intensity of migraine attacks during weeks 5-8 were significantly lower in the Shaoyang-specific acupuncture group than in the control group. During weeks 13-16, patients in all three treatment groups reported significantly fewer days with a migraine compared to patients in the control group (Table 3 ).

Patients in both Shaoyang acupuncture groups reported better migraine-specific quality of life compared with patients in the control group. Few significant differences were observed between patients in the three acupuncture groups for this outcome (Table 3).

When we performed the sensitivity analyses after the imputation of missing data and evaluating the per-protocol group, we obtained results similar to those obtained in our main analysis.

\section{Safety}

In total, 37 patients $(7.8 \%)$ experienced 42 adverse events during the study period (9 in the Shaoyang-specific acupuncture group, 8 in the

Table 2: Baseline characteristics of 476 patients who received acupuncture or sham acupuncture

No. (\%) of patients*

\begin{tabular}{|c|c|c|c|c|c|}
\hline \multirow[b]{2}{*}{ Characteristic } & \\
\hline & $\begin{array}{l}\text { Shaoyang- } \\
\text { specific } \\
\text { acupuncture } \\
n=121\end{array}$ & $\begin{array}{c}\text { Shaoyang- } \\
\text { nonspecific } \\
\text { acupuncture } \\
n=119\end{array}$ & $\begin{array}{c}\text { Yangming-specific } \\
\text { acupuncture } \\
n=118\end{array}$ & $\begin{array}{c}\text { Sham acupuncture } \\
\text { (control) } \\
n=118\end{array}$ & $\begin{array}{l}\text { Overall } \\
n=476\end{array}$ \\
\hline Women & $100(82.6)$ & $99(83.2)$ & $92(78.0)$ & $103(87.3)$ & $394(82.8)$ \\
\hline Age, yr, mean (SD) & $37.1(11.7)$ & $36.2(12.4)$ & $36.8(13.0)$ & $37.5(12.1)$ & $36.9(12.3)$ \\
\hline \multicolumn{6}{|l|}{ Migraine type } \\
\hline With aura & $18(14.9)$ & $14(11.8)$ & $12(10.2)$ & $12(10.2)$ & $56(11.8)$ \\
\hline Without aura & $103(85.1)$ & $105(88.2)$ & $106(89.8)$ & $106(89.8)$ & $420(88.2)$ \\
\hline Duration of illness, mo, mean (SD) & $119.8(115.3)$ & $91.8(78.6)$ & $104.0(100.7)$ & $102.0(93.4)$ & $98.1(5.0)$ \\
\hline \multicolumn{6}{|l|}{$\begin{array}{l}\text { Self-reported migraine intensityt on a } \\
\text { scale of } 0-3\end{array}$} \\
\hline Mild (1) & $24(20.0)$ & $14(11.8)$ & $15(12.7)$ & $17(14.4)$ & $70(14.7)$ \\
\hline Moderate (2) & $74(61.0)$ & $80(67.2)$ & $87(73.7)$ & $81(68.6)$ & $322(67.6)$ \\
\hline Severe (3) & $23(19.0)$ & $16(21.0)$ & $16(13.6)$ & $20(17.0)$ & $84(17.7)$ \\
\hline Previous use of acupuncture & $5(4.1)$ & $2(1.6)$ & $1(0.8)$ & 4 (3.3) & $12(2.5)$ \\
\hline Use of acute pain medication & $35(28.9)$ & 40 (33.6) & $36(30.5)$ & $45(38.1)$ & $156(32.8)$ \\
\hline Use of analgesics & $20(16.5)$ & 19 (15.9) & $15(12.7)$ & $25(21.2)$ & $79(16.6)$ \\
\hline Ergotamine & $1(0.8)$ & $2(1.7)$ & $3(2.5)$ & $0 \quad(0.0)$ & $6(1.3)$ \\
\hline $\begin{array}{l}\text { Traditional Chinese medicine (herbs, } \\
\text { pills or capsules) }\end{array}$ & $14(11.6)$ & $15(12.6)$ & $15(12.7)$ & $17(14.4)$ & $61(12.8)$ \\
\hline Combination & $0(0.0)$ & $4(3.4)$ & $3(2.5)$ & $3(2.5)$ & $10(2.1)$ \\
\hline $\begin{array}{l}\text { Note: SD = standard deviation. } \\
\text { *Unless otherwise stated. } \\
\text { tDuring a four-week period before treatment. }\end{array}$ & & & & & \\
\hline
\end{tabular}




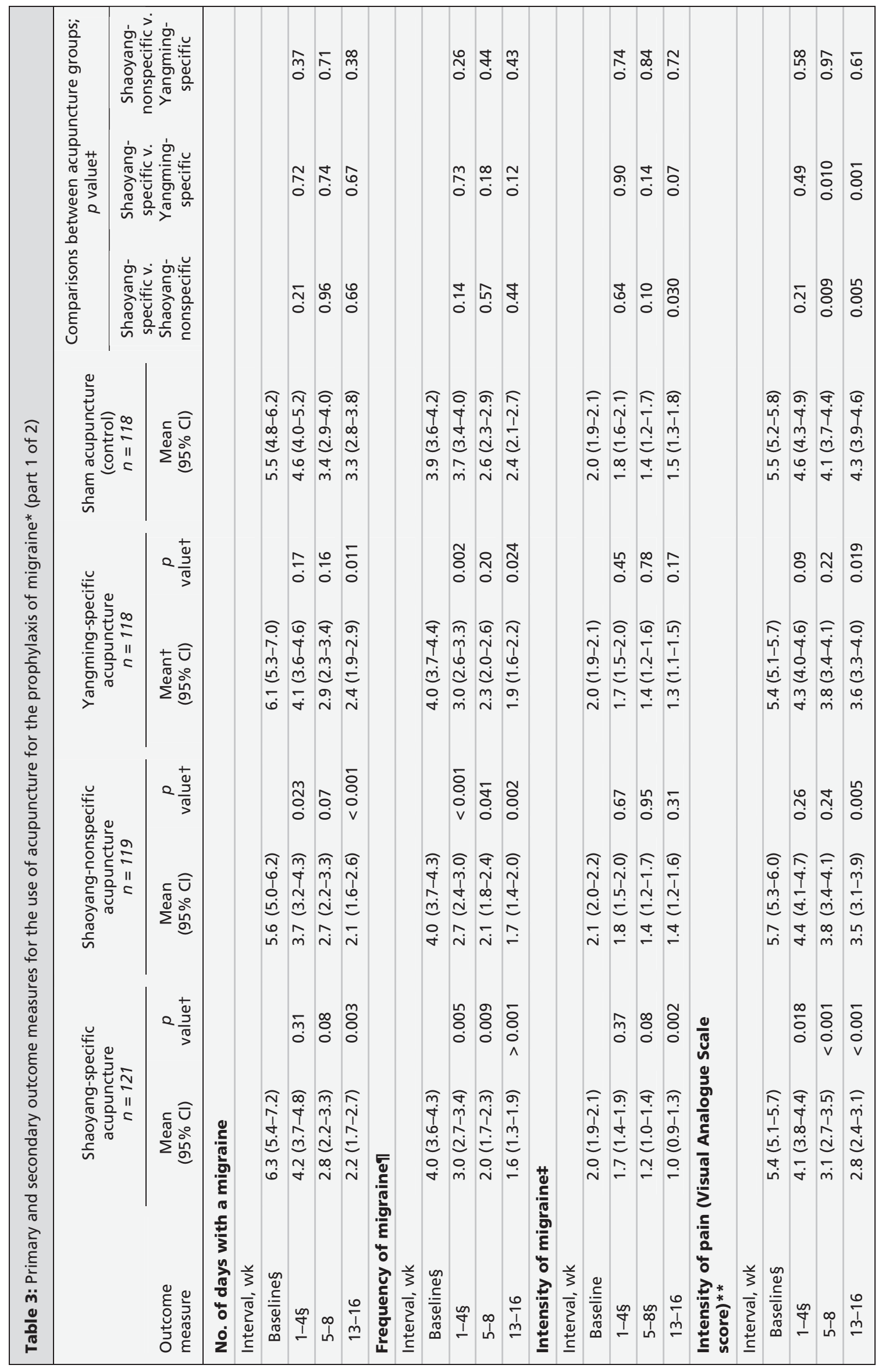




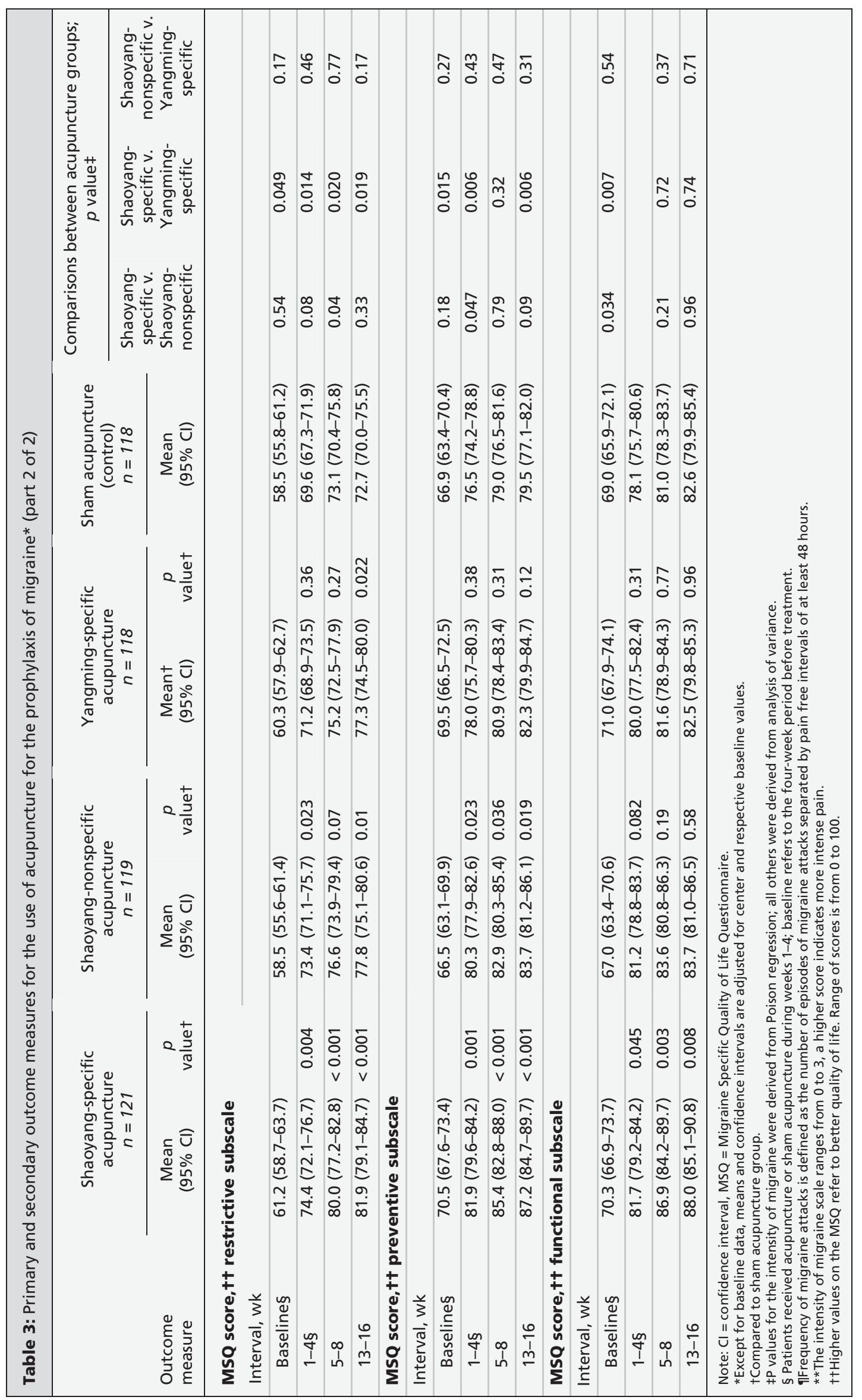


Shaoyang-nonspecific acupuncture group, 12 in the Yangming-specific acupuncture and 8 in the control group). Subcutaneous hemorrhage was the most common adverse effect ( 25 patients [67.6\% of patients who experienced an adverse event]; 6 in the Shaoyang-specific acupuncture group, 5 in the Shaoyang-nonspecific acupuncture group, 10 in the Yangming-specific acupuncture group and 4 in the control group), followed by subcutaneous hematoma (6 patients [16.2\% of patients who experienced an adverse event]; 1 in the Shaoyang-specific group, 3 in the Shaoyang-nonspecific acupuncture group, 2 in the Yangming-specific acupuncture group), and subcutaneous ecchymosis (5 patients [13.5\% of patients who experienced an adverse event], 1 in the Shaoyang-specific acupuncture group and 4 in the control group). One patient (2.7\% of patients who experienced an adverse events) in the Shaoyang-specific acupuncture group reported leg weakness. All participants recovered fully from the adverse events.

\section{Interpretation}

We found that acupuncture was more effective than sham acupuncture for almost all secondary outcomes during both study periods (weeks 5-8 and 13-16 after randomization). There was no difference in the number of days with a migraine during the four-week period after treatment. However, we found a clinically minor effect after 16 weeks. We found no relevant differences between the three acupuncture groups.

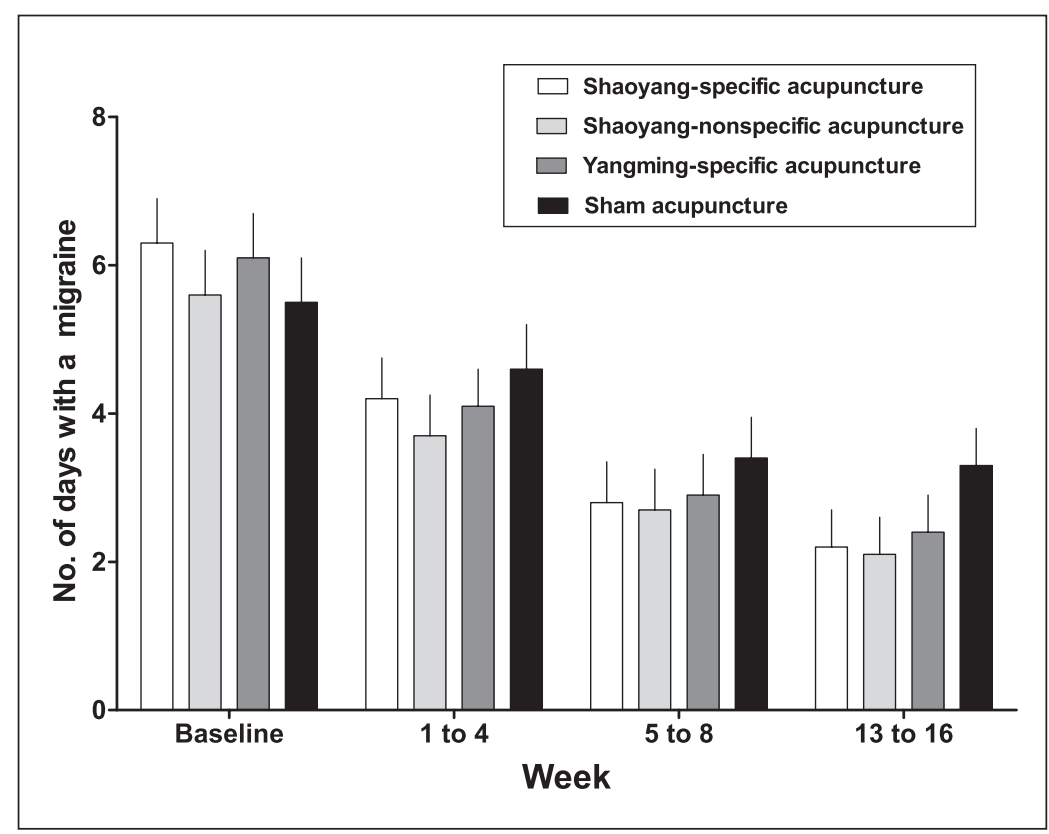

Figure 2: Mean (95\% confidence intervals) number of days with a migraine. during the study period. Baseline data was collected during a four-week period before acupuncture began.
Our secondary findings must be interpreted with caution because of the exploratory test statistics. Our trial had a large sample size, a prevalidated sham control, rigorous experimental methods (including blinding, central randomization, standardization of the intervention, prepublished study protocol, ${ }^{19}$ predefined statistical analysis and independent data analyses by two statisticians.

Our results indicate that the style of acupuncture has little relevance on the outcome. Shaoyang-specific acupuncture points did not result in better outcomes than other acupuncture points, suggesting that point-specific effects play a small role in the overall effect. According to our results, nonspecific effects (e.g., expectations and patient-practitioner interaction) may have had a more prominent role and been increased by the use of electrostimulation in all groups. However, the use of electrostimulation is typical in China and was used in the control group to ensure blinding. Some types of electrostimulation have been reported to be beneficial for short-term relief of pain, ${ }^{27-29}$ potentially leading to stronger pain-control mechanisms than intended in the control group. This might be explained by the endorphin hypothesis (release of endogenous opioids by electrostimulation). Melzack's gate control theory and diffuse noxious inhibitory control might explain the stronger effect on pain inhibitory mechanisms caused by more intense local pain stimulus induced by electrostimulation. ${ }^{30-33}$ This might explain why we found no significant differences between the acupuncture and sham acupuncture groups directly following the treatment period (weeks 5-8) but we did find differences later (weeks 13-16). Based on results from previous trials involving patients with migraines, ${ }^{8,11,12,34}$ we speculate that the effect would have increased even further with a longer follow-up period. The higher frequency and number of treatments used in our trial (compared with other Western studies ${ }^{11,12}$ ) did not result in clinically meaningful effects at the end of the intervention, and the results of our study could be difficult to generalize to Western populations.

A recent Cochrane review of acupuncture for migraine prophylaxis ${ }^{6}$ concluded that no evidence exists for an effect from "true" acupuncture compared with sham interventions, but there was an effect compared with usual care. However, we found that the outcomes following acupuncture were significantly better than with sham acupuncture during weeks 13-16, but the effect was clinically minor. Of the two largest acupuncture trials included in the Cochrane review, ${ }^{10,11}$ the patients included in our trial and our results are more comparable with the trial by Diener and colleagues, ${ }^{11}$ in 
which the patients had a similar mean number of migraine attacks and days with migraine. However, our results had less variance. Diener and colleagues also reported a significant, but clinically minor, difference between acupuncture and sham acupuncture at follow-up (26 wks) but not at the end of treatment (13 wks).

The fact that only one-third of patients in our trial used acute pain medication at baseline is in line with the results from a cross-sectional study in China, which showed that only half of the outpatient of a neurologic department used analgesics for the treatment of migraine. ${ }^{35}$

\section{Limitations}

The limitations of our trial include a short follow-up period and self-reported outcome measures. In addition, the physicians were not blinded as to the patients' treatment assignment. Thus, we are unsure of how much of the observed changes represent the biological effects of acupuncture and how much is due to nonspecific effects. However, patients were unaware of their treatment group, and all had been informed that they would receive "real" acupuncture in order to reduce the chance of unblinding.

Patients in the Shaoyang-specific acupuncture group reported the highest number of days with a migraine at baseline and the greatest effect after treatment, but these analyses were based on analysis of covariance models with baselineadjustments, which accounts, to some extent, for the problem of regression to the mean.

Although there is a current trend toward comparative effectiveness research, ${ }^{36-38}$ our trial had a strong focus on efficacy.

The small number of acupuncture points included in our trial might be another limitation; however, the points that we chose are commonly used and have been shown to be beneficial for the treatment of acute migraine attacks. ${ }^{23}$

\section{Conclusion}

Acupuncture appeared to have a clinically minor prophylactic effect for migraine. However, the nonspecific effects of acupuncture may play a relevant role, and future research should provide more insight into the nature of these effects.

\section{References}

1. Steiner TJ, Scher AI, Stewart WF, et al. The prevalence and disability burden of adult migraine in England and their relationships to age, gender and ethnicity. Cephalalgia 2003;23:519-27.

2. Lipton RB, Diamond S, Reed M, et al. Migraine diagnosis and treatment: results from the American Migraine Study II Headache 2001;41:638-45.

3. Guo S. Preliminary investigation on the epidemiology of migraine in China [article in Chinese]. Zhonghua Liu Xing Bing Xue Za Zhi 1993;14:102-5.

4. Cheung RT. Prevalence of migraine, tension-type headache, and other headaches in Hong Kong. Headache 2000;40:473-9.
5. Wang SJ, Fuh JL, Young YH, et al. Prevalence of migraine in Taipei, Taiwan: a population-based survey. Cephalalgia 2000; 20:566-72.

6. Linde K, Allais G, Brinkhaus B, et al. Acupuncture for migraine prophylaxis. Cochrane Database Syst Rev 2009;CD001218.

7. Liang FR. The 973 Project: research of specificity of acupuncture points based on clinical studies. Journal of Chengdu University of Traditional Chinese Medicine 2007;30:1-2.

8. Facco E, Liguori A, Petti F, et al. Traditional acupuncture in migraine: a controlled, randomized study. Headache 2008; 48 : 398-407.

9. Alecrim-Andrade J, Maciel-Junior JA, Carne X, et al. Acupuncture in migraine prevention: a randomized sham controlled study with 6-months posttreatment follow-up. Clin J Pain 2008;24:98-105.

10. Linde K, Streng A, Jurgens S, et al. Acupuncture for patients with migraine: a randomized controlled trial. JAMA 2005;293: 2118-25.

11. Diener HC, Kronfeld K, Boewing G, et al. Efficacy of acupuncture for the prophylaxis of migraine: a multicentre randomised controlled clinical trial. Lancet Neurol 2006;5:310-6.

12. Alecrim-Andrade J, Maciel-Junior JA, Cladellas XC, et al Acupuncture in migraine prophylaxis: a randomized sham-controlled trial. Cephalalgia 2006;26:520-9.

13. Linde M, Fjell A, Carlsson J, et al. Role of the needling per se in acupuncture as prophylaxis for menstrually related migraine: a randomized placebo-controlled study. Cephalalgia 2005;25:41-7.

14. You Z. Jinkuiyi. Shanghai (China): Health publisher; 1957.

15. Lingshu. Beijing (China): People's Medical Publishing House; 1957.

16. Chen $\mathrm{Q}, \mathrm{Wu} \mathrm{X}, \mathrm{Zhu} \mathrm{H}$, et al. Analysis of acupuncture point used in clinical controlled trials of acupuncture for migraineurs. J Chengdu Univ Trad Chinese Med 2007;30:1-9.

17. Tfelt-Hansen P, Block G, Dahlof C, et al. Guidelines for controlled trials of drugs in migraine: second edition. Cephalalgia. 2000;20:765-86

18. Zhao L, Liang FR, Li Y, et al. Improved quality monitoring of multi-center acupuncture clinical trials in China. Trials 2009; 10:123.

19. Jena S, Witt CM, Brinkhaus B, et al. Acupuncture in patients with headache. Cephalalgia 2008;28:969-79.

20. Li Y, Liang F, Yu S, et al. Randomized controlled trial to treat migraine with acupuncture: design and protocol. Trials 2008;9:57.

21. Headache Classification Subcommittee of the International Headache Society. The International Classification of Headache Disorders: 2nd edition. Cephalalgia. 2004;24 Suppl 1:9-160.

22. Yang XG, Li Y, Tian XP, et al. Location of sham acupuncture points in acupuncture studies: a systematic review. [article in Chinese]. J Tradit Chin Med 2009;50:748-50.

23. Li Y, Liang FR, Yang X, et al. Acupuncture for treating acute attacks of migraine: a randomized controlled trial. Headache 2009; $49: 805-16$

24. Jhingran P, Osterhaus JT, Miller DW, et al. Development and validation of the Migraine-Specific Quality of Life Questionnaire. Headache 1998;38:295-302.

25. Cole JC, Lin P, Rupnow MF. Validation of the Migraine-Specific Quality of Life Questionnaire version 2.1 (MSQ v. 2.1) for patients undergoing prophylactic migraine treatment. Qual Life Res 2007; 16:1231-7.

26. Cole JC, Lin P, Rupnow MF. Minimal important differences in the Migraine-Specific Quality of Life Questionnaire (MSQ) version. Cephalalgia 2009;29:1180-7.

27. Niddam DM. Brain manifestation and modulation of pain from myofascial trigger points. Curr Pain Headache Rep 2009;13:370-5.

28. Nekhendzy V, Lemmens HJ, Tingle M, et al. The analgesic and antihyperalgesic effects of transcranial electrostimulation with combined direct and alternating current in healthy volunteers Anesth Analg 2010;111:1301-7.

29. Gabis L, Shklar B, Baruch YK, et al. Pain reduction using transcranial electrostimulation: a double blind "active placebo" controlled trial. J Rehabil Med 2009;41:256-61.

30. Melzack R, Wall PD. Pain mechanisms: a new theory. Science 1965;150:971-9.

31. Carlsson C. Acupuncture mechanisms for clinically relevant long-term effects - reconsideration and a hypothesis. Acupunct Med 2002;20:82-99.

32. Rossi P, Serrao M, Perrotta A, et al. Neurophysiologica approach to central pain modulation in primary headaches. $J$ Headache Pain 2005;6:191-4.

33. Endres HG, Diener HC, Molsberger A. Role of acupuncture in the treatment of migraine. Expert Rev Neurother 2007;7:1121-34

34. Alecrim-Andrade J, Maciel-Júnior J, Cladellas X, et al Acupuncture in migraine prophylaxis: a randomized shamcontrolled trial. Cephalalgia 2006;26:520-9.

35. Wang Y, Zhou J, Fan X, et al. Classification and clinical features 


\section{RESEARCH}

of headache patients: an outpatient clinic study from China. $J$ Headache Pain 2011;12:561-7.

36. Conway PH, Clancy C. Comparative-effectiveness research implications of the Federal Coordinating Council's report. $N$ Engl J Med 2009;361:328-30.

37. Garber AM, Tunis SR. Does comparative-effectiveness research threaten personalized medicine? N Engl J Med 2009;360:1925-7.

38. Schumock GT, Pickard AS. Comparative effectiveness research: relevance and applications to pharmacy. Am J Health Syst Pharm 2009;66:1278-86.

Affiliations: From the Chengdu University of Traditional Chinese Medicine (Li, Zheng, Yu, Zhao, Huang, Liang), Chengdu, Sichuan, China; the Institute for Social Medicine (Witt, Roll, Huang), Epidemiology and Health Economics, Charité University Medical Center, Berlin, Germany; the Center for Integrative Medicine (Witt), University of Maryland School of Medicine, Baltimore, Md.; the Hunan University of Traditional Chinese Medicine (Yan, Chang, Lan), Changsha, Hunan, China; and Hubei University of Traditional Chinese Medicine (Sun, Zhang, Zou), Wuhan, Hubei, China.

Contributors: Ying Li had full access to all aspects of this trial and was responsibile for the implementation and supervision of the study. Ying Li, Fan-rong Liang, Shu-guang Yu, Wen-jing Huang, Jie Yan, Guo-jie Sun and Xiao-rong Chang contributed to the study concept and design. Methodologic advice and statistical analysis were provided by Claudia Witt and Stephanie Roll. Data were acquired by Hui Zheng, Ying Li. Hui Zheng, De-jun Wang and Lei Lan. Ran Zou managed the clinical monitoring. Study implementation and supervision were managed by Ling Zhao, Ying Li, Fan-rong Liang, Jie Yan, Guo-jie Sun and Xiao-rong Chang. Ying Li and Hui Zheng drafted the manuscript. They, along with all other authors, revised the manuscript for important content. Administrative, technical or material support was provided by Shu-guang Yu, Hong-xing Zhang, Jie Yan, Guo-jie Sun and Xiao-rong Chang. Funding was obtained by Fan-rong Liang.

Funding: This trial was funded by the National Basic Research Program of China (973 Program, no. 2006CB504501). The sponsor had no role in the design of the study, the collection, analysis or interpretation of the data, the writing of the manuscript or the decision to submit the article for publication.

\section{Participating trial centres}

- First affiliated hospital of Chengdu University of Traditional Chinese Medicine

- People's Hospital of Sichuan Province

- No. 4 People's Hospital of Sichuan Province

- Affiliated Hospital of Ningxia Medicine University

- First affiliated Hospital of Hunan University of Traditional Chinese Medicine

- People's Hospital of Chenzhou City

- Shekou Traditional Chinese Medicine hospital of Shenzhen City

- No.1 People's Hospital of Wuhan City

- Wuhan Hospital of Traditional Chinese Medicine 